\title{
Photoluminescence measurements of zero-phonon optical transitions in silicon nanocrystals
}

\author{
I. Sychugov, ${ }^{1,3,5}$ J. Valenta, ${ }^{2}$ K. Mitsuishi, ${ }^{3}$ M. Fujii, ${ }^{4}$ and J. Linnros ${ }^{5}$ \\ ${ }^{1}$ ICYS, National Institute for Materials Science, Sengen 1-2-1, Tsukuba, 3050047 Japan \\ ${ }^{2}$ Department of Chemical Physics and Optics, Faculty of Mathematics and Physics, Charles University, Ke Karlovu 3, Prague 2, \\ CZ-12116 Czech Republic \\ ${ }^{3}$ High Voltage Electron Microscopy Station, National Institute for Materials Science, Sakura 3-13, Tsukuba, 3050003 Japan \\ ${ }^{4}$ Department of Electrical and Electronic Engineering, Graduate School of Engineering, Kobe University, 1-1 Rokkodai, Nada, Kobe, \\ 6578501 Japan \\ ${ }^{5}$ Department of Material Physics, School of ICT, Royal Institute of Technology, Electrum 229, Kista-Stockholm, SE-16440 Sweden \\ (Received 6 April 2011; revised manuscript received 5 July 2011; published 30 September 2011)
}

\begin{abstract}
Optical transitions in silicon nanocrystals with different surface passivations were probed at low temperatures on a single-particle level. A type of quasidirect recombination process, different from the quantum-confined exciton transition, is identified. The luminescence spectra have different emission energies, but the contribution of a no-phonon transition is significantly higher than expected from the quantum-confinement model. Its relative strength was found to be temperature dependent, suggesting spatial localization of excitons as a possible origin.

DOI: 10.1103/PhysRevB.84.125326

PACS number(s): 78.67.Bf, 78.55.Ap, 78.67.Hc
\end{abstract}

\section{INTRODUCTION}

Silicon is an indirect band-gap material, i.e., participation of a momentum-conserving phonon is required for optical transitions from an excited to the ground state. Indeed, the transverse-optical (TO) phonon-assisted line dominates the low-temperature luminescence spectrum of bulk silicon. ${ }^{1}$ Consequently, bulk $\mathrm{Si}$ is an inefficient light emitter, although it is widely used for the detection (charge-coupled devices and diodes) of electromagnetic radiation. Therefore, the practical realization of integrated optical circuits requires an external light source. Typically, it is composed of a direct band-gap material, e.g., III-V group semiconductors ${ }^{2}$ or doped and strained Ge (Ref. 3).

The poor light-emitting properties of $\mathrm{Si}$ are, however, much improved in small nanocrystals (NCs) due to the effect of quantum confinement (QC). When the size of a silicon structure is less than the exciton Bohr diameter $(\sim 10 \mathrm{~nm})$, the wave functions of spatially confined carriers experience a strong overlap in $k$ space, leading to the optical transition not existing in pure bulk material. This recombination channel is different from the truly direct recombination where carrier positions in $k$ space match. ${ }^{4}$ The rate of this quasidirect transition depends on the NC size, and, in principle, can substantially be increased for small NCs. ${ }^{5}$ Experimentally, this size-dependent recombination channel was detected in ensemble measurements. ${ }^{6}$

Single-dot spectroscopy is, however, a better optical tool, avoiding the inherent inhomogeneity of ensemble studies. Previously, we applied it to low-temperature (down to $35 \mathrm{~K}$ ) measurements of Si NCs prepared by self-limiting oxidation of nanopillars. ${ }^{7}$ A narrow luminescence linewidth and various emission energies were observed in support of the QC nature of the carrier recombination. However, the behavior of a TO-phonon line (TOL) in the recorded spectra deviated from the QC model prediction. Instead of a size-dependent reduction in its intensity in favor of the zero-phonon line (ZPL), a general strong dominance of the ZPL was found. To address this issue, here, we have carried out experiments targeting single Si NCs with different passivations. It became possible due to the development of a different lithographical technique for nanostructure preparation described in Ref. 8. The NCs were fabricated from the top layer of silicon-on-insulator (SOI) wafers by lithography, etching, and short oxidation.

In this paper, we analyze results of low-temperature singledot spectroscopic studies on Si nanocrystals prepared by rapid and self-limiting oxidation with comparison to the QC theory. An improved optical setup was used to detect low-intensity luminescence down to $10 \mathrm{~K}$ (Ref. 9), and a large number of spectra was recorded for statistical analysis. As a result, we identify a different type of quasidirect optical transition in Si NCs. This recombination channel is found to be size independent but temperature sensitive and is ascribed to a localized state-mediated transition.

\section{EXPERIMENTAL DETAILS}

Samples were prepared from $n$-type low-doped waferbonded $\langle 100\rangle$ SOI wafers with a $1.1-\mu \mathrm{m}$ buried oxide and a 7.5- $\mu \mathrm{m}$ top $\mathrm{Si}$ layer. The silicon film was reduced by $\mathrm{SF}_{6}$ etching to an ultrathin layer in a plasma reactor. When the chip edges already reveal the surface of the buried $\mathrm{SiO}_{2}$, the silicon layer still remains in the middle of the sample. Then, the borderline region is only a few-nanometers-thick silicon film. ${ }^{8}$ The sub-10-nm metal mask was formed on this region by electron-beam-induced deposition of tungsten. ${ }^{10}$ After a second plasma etching of silicon, the metal mask was selectively removed by a heated aqua regia solution. Finally, the samples went through rapid thermal annealing in a pure $\mathrm{O}_{2}$ atmosphere at $900^{\circ} \mathrm{C}$. The oxidation time was very short, from 10 to $30 \mathrm{~s}$, resulting in a few-nanometers oxide passivation. Longer oxidation was found to completely consume the $\mathrm{Si}$ NCs. This situation is different from the self-limiting oxidation used for the fabrication of NCs in individual nanopillars. In that case, a several-hours long oxidation was employed, resulting in a thicker oxide as well as inherent interfacial stress. ${ }^{11}$ Low-temperature photoluminescence (PL) studies of the SOI nanodots were carried out with 405-nm laser excitation in an epifluorescence configuration. The sample was placed in a cryostat, and the signal was collected by a $40 \times$ objective 


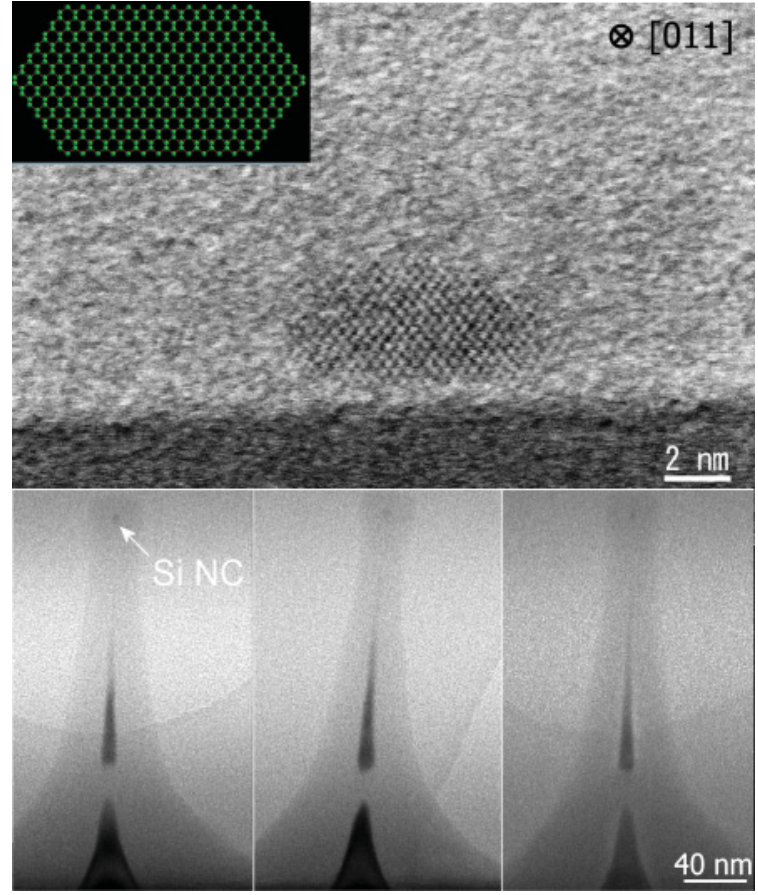

FIG. 1. (Color online) (Top) Cross-sectional bright-field scanning TEM image of an Si NC formed on top of an SOI wafer. It resides on the buried oxide and is surrounded by the thermally grown passivating $\mathrm{SiO}_{2}$ shell. The inset shows a 3D model of an $\mathrm{Si} \mathrm{NC}$ oriented along the [011] direction with the same dimensions consisting of $\sim 8000$ atoms. (Bottom) Cross-sectional TEM images of three different oxidized $\mathrm{Si}$ nanopillars. As an effect of self-limiting oxidation, an NC can be seen at the top of these nanostructures.

lens with a numerical aperture of 0.6. The typical acquisition time for a single spectrum was $30 \mathrm{~min}$. For cross-sectional transmission electron microscope (TEM) imaging, a focused ion-beam preparation of the patterned area with a carbon protection layer was used.

To compare NCs fabricated by the present method with NCs located in pillars fabricated using our previous method, ${ }^{7}$ their cross-sectional TEM images are presented in Fig. 1. A high-resolution image of an SOI nanodot from a patterned array is shown in Fig. 1, top. The oxidation time of the sample was $30 \mathrm{~s}$, and the image was taken along the [011]-zone axis. It is seen that a several-nanometers-thick $\mathrm{SiO}_{2}$ shell surrounds the Si NC. The dark lower part is the buried oxide of the SOI wafer. The inset shows a three-dimensional (3D) simulation of a silicon $\mathrm{NC}$ with the same orientation and dimensions $(7 \times 7 \times 4 \mathrm{~nm})$, assuming a symmetrical nanodot geometry. It consists of about 8000 atoms and has faceting along the $\{111\}$-crystallographic planes. Below on the same figure, three different oxidized nanopillars, with isolated Si NCs on top, are shown. As can be seen, here, the oxide layer is about $15-20 \mathrm{~nm}$, which is thicker than for SOI nanodots as a result of long self-limiting oxidation.

It is important to note that, along with patterned structures, random objects also exist in these samples. They can be formed when a surface particle acts as a mask for etching or due to uneven oxidation of the thin Si layer. The inset in Fig. 2 shows a PL image of the SOI nanodot sample, where single objects can be seen along with a bright area nearby. The emission from

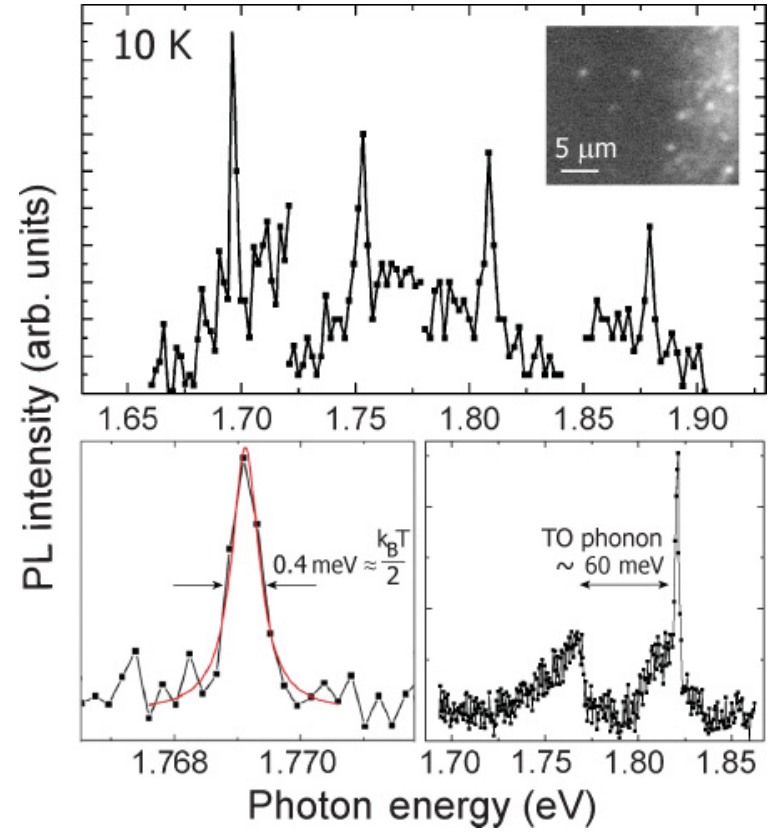

FIG. 2. (Color online) (Top) Four PL spectra recorded at $10 \mathrm{~K}$ from a sample with SOI nanodots, featuring different emission wavelengths from different objects. A PL image is also shown where single emitters can be discerned. (Bottom) Spectra exhibiting (left) a narrow linewidth and (right) a several-meV phonon and TO-phonon sidebands.

the bright region is attributed to luminescence from Si quantum wells (QWs). A PL spectrum from this region was found to be temperature independent with two broad peaks centered at 1.4 and $1.75 \mathrm{eV}$, similar to the one reported earlier for Si QWs. ${ }^{12}$ Coexistence of crystalline nanodots and nanolayers of $\mathrm{Si}$ in the borderline region of the film was confirmed by cross-sectional and plane-view TEM imaging.

\section{RESULTS}

We start the results discussion with low-temperature PL data taken from the new batch at $10 \mathrm{~K}$. Despite the difference in preparation routine, the line shapes appear to be the same as for oxidized nanopillars. In Fig. 2, several spectra from different dots are shown where the two in the bottom panels were recorded with a higher spectral resolution. It is seen that peak positions are different, while the deconvoluted linewidth of $\sim 400 \mu \mathrm{eV}$ is actually the narrowest ever measured for an $\mathrm{Si}$ nanostructure (cf. $k_{B} T=860 \mu \mathrm{eV}$ at $10 \mathrm{~K}$ ). The variation in the peak position and a smaller than thermal broadening linewidth are characteristics of a single-dot emission subject to QC. It is a practical manifestation of the size-dependent discrete energy states of a quantum dot. ${ }^{13}$ Some of the recorded spectra exhibit a broader linewidth, which most likely can be attributed to spectral diffusion or scattering events averaged over the long acquisition time. ${ }^{14}$ A several-meV sideband can be discerned in better signal-to-noise ratio spectra (Fig. 2, bottom right). It was also detected in the luminescence of NCs in nanopillars and was attributed to acoustic phonon modes specific to NCs. ${ }^{15}$ These modes correspond to stretching or twisting of the NC as calculated in Ref. 16. In addition, this spectrum reveals an $\mathrm{Si}$ TO-phonon replica, which is observed only in a few spectra at 


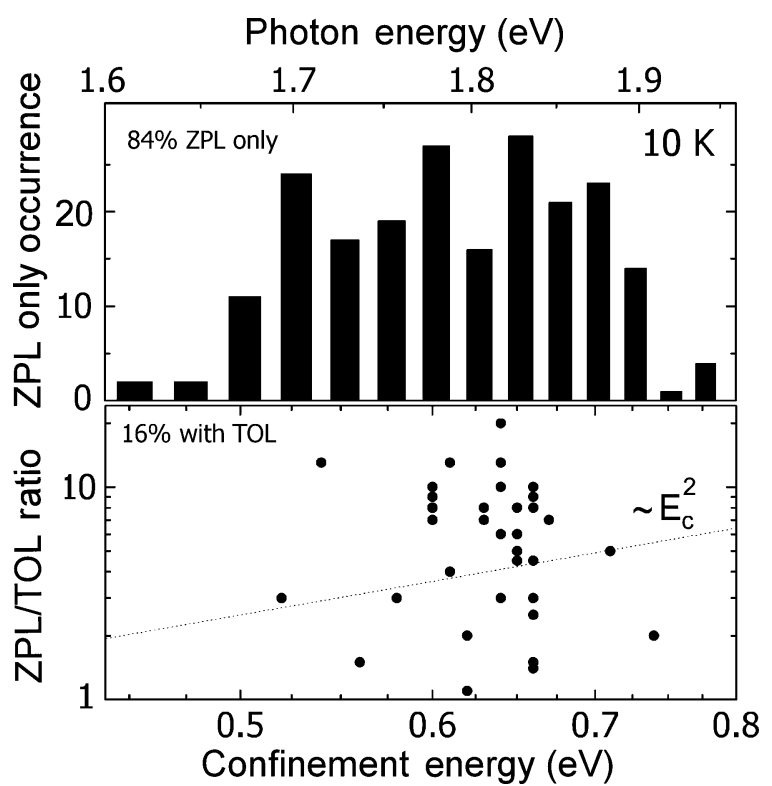

FIG. 3. (Top) Histogram of ZPL-only spectra measured at $10 \mathrm{~K}$ for SOI nanodots; (bottom) ZPL/TOL intensity ratio as a function of the confinement energy for the remaining $16 \%$ of the spectra featuring a TO-phonon replica. The broken line is a QC model prediction.

this temperature. With increasing temperature, the line shape broadens, and the TO-phonon satellite appears in more spectra, as discussed below.

The involvement of the TO-phonon line in the emission band at $10 \mathrm{~K}$ is summarized in Fig. 3. The majority of recorded spectra have a ZPL-only line shape (Fig. 3, top). The remaining spectra, featuring both ZPL and TOL peaks, comprise about $16 \%$ of the data set. The ZPL/TOL ratio for these spectra is shown below as a function of the confinement energy $E_{\mathrm{c}}=h v-E_{\mathrm{gap}}^{\mathrm{Si}}$, where $E_{\mathrm{gap}}^{\mathrm{Si}}=1.16 \mathrm{eV}$. The ratio is more than unity, and the data points are randomly scattered. The same data pattern for TOL spectra was observed for all temperatures and samples investigated here. Recently, a similar random ZPL/TOL ratio distribution also was reported for single Si nanorods at $80 \mathrm{~K} .{ }^{17} \mathrm{On}$ the other hand, the prediction of the QC model would be a general power-law dependence $\sim E_{c}^{2}$ (Ref. 6), shown as a broken line in Fig. 3 (here, the proportionality coefficient was chosen to accommodate present data and, thus, was larger than in the original paper). In the QC model, the general dependence of the ZPL strength on the confinement energy follows from the increasing degree of particle wave-function overlap in smaller NCs.

The results at various temperatures are presented in Fig. 4. As a statistical measure of the TO-phonon-assisted transition strength, the fraction of spectra with a TOL is shown. It is seen that this fraction grows with temperature for both samples. The trend was noticed in the previous paper yet was left unexplained. ${ }^{7}$ Here, we discuss the problem of phonon involvement in the recombination process by considering different kinds of exciton localizations.

\section{DISCUSSION}

In general, zero-phonon transitions are forbidden in silicon where excited carriers occupy different positions in $k$ space:

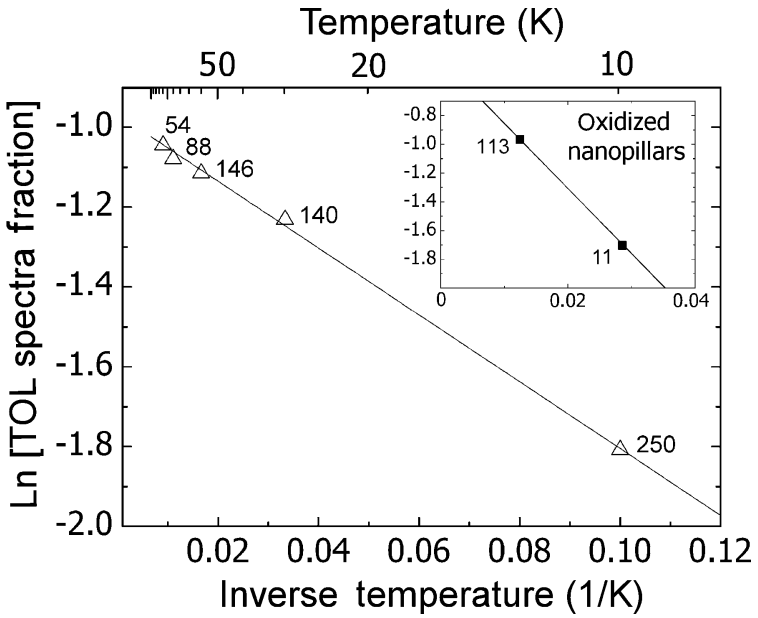

FIG. 4. Arrhenius plot of the fraction of spectra exhibiting TOL versus temperature for SOI nanodots (open triangles) and oxidized nanopillars (solid squares, inset). Numbers are total spectra recorded. The solid line is a fit based on a single rate-limited thermally deactivated process.

close to the $X$ point for an electron and at the $\Gamma$ point for a hole. ${ }^{18}$ However, the localization of carriers in real space increases their momentum uncertainty, following Heisenberg's relation $\Delta p \Delta x \sim \hbar$, where $\Delta p=\hbar \Delta k .{ }^{19}$ We consider two types of exciton localization, leading to the appearance of a zero-phonon line: confinement in a potential well and binding to the lattice irregularity. In the case of QC, the carriers are localized inside a potential well of size $L$. Then, particles cannot be treated as points on the band diagram, but instead, the corresponding wave functions have a spread in $k$ space on the order of $1 / L .^{5}$ The resulting wave-function overlap allows carrier interaction without phonon mediation. This effect depends on the NC size, where a stronger process is expected in small NCs. ${ }^{5}$ The wave function of a spatially localized particle, on the other hand, can be described as exponentially decaying within a characteristic length $l:|\psi(r)|^{2} \sim \exp \left(-r^{2} / l^{2}\right){ }^{20}$ In $k$ space, such a damped wave has a coefficient $\exp \left(-k^{2} l^{2} / 2\right)$, implying that $k$ eigenvalues also have a finite linewidth on the order of $1 / l$. In practice, this type of breakdown of the $k$-conservation rule is observed in bulk $\mathrm{Si}$ as a no-phonon recombination of the exciton bound to an impurity center. ${ }^{1}$ In both cases, the contribution of localization to the particle energy is $\Delta E=(\hbar \Delta k)^{2} / 2 m^{*}$, where $m^{*}$ is a particle effective mass. ${ }^{19}$

Thus, both the detected photon energy and the strength of the no-phonon line are defined by the type of localization. An exciton bound to a lattice irregularity has a fixed energy, while a size-dependent QC effect results in different emission energies, as observed here. On the other hand, the dominance of ZPL at low temperatures and the lack of its relative strength dependence on the emission energy (i.e., NC size) are indicative of the exciton-binding mechanism. So, it is reasonable to suggest that both effects take place in Si NCs, and their possible interplay is discussed below.

The fraction of ZPL-only spectra gradually reduces with temperature (Fig. 4), suggesting that the contribution of the localized state is a function of temperature. Thus, using an 


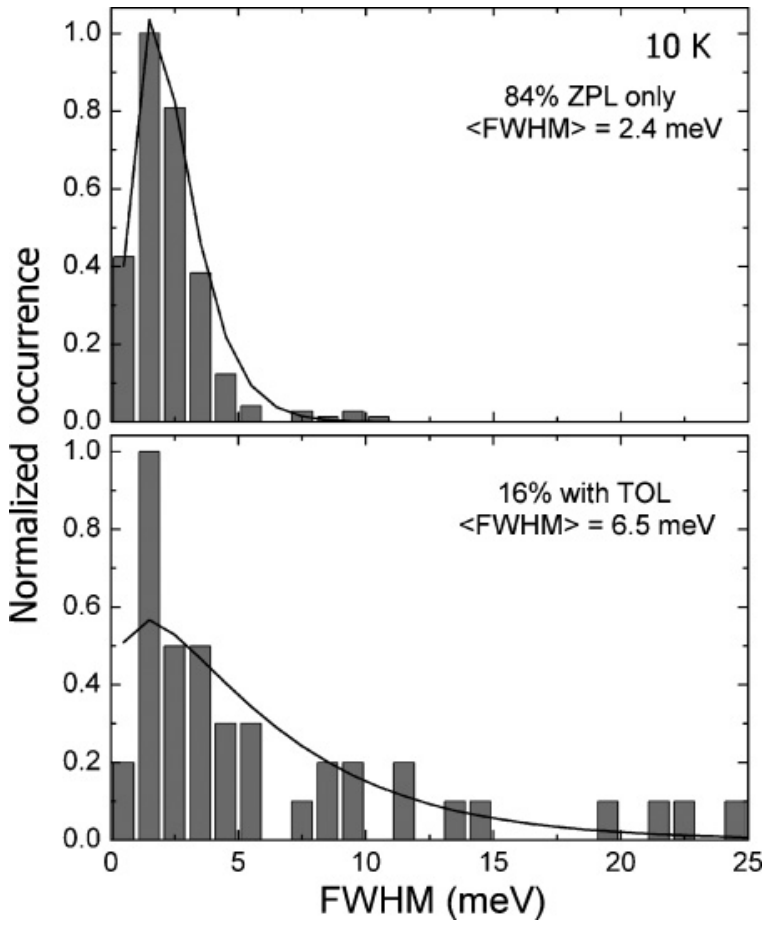

FIG. 5. Histograms of the main peak full width at half maximum (FWHM) measured at $10 \mathrm{~K}$ for SOI nanodots for (top) zero-phonon line shapes and (bottom) the remaining spectra with a TO-phonon replica. The solid lines are guides for the eye only. Also, average values are shown.

analogy of the bound exciton in bulk Si subject to thermal delocalization, ${ }^{21}$ a localized state with a probability of thermal deactivation can be considered. Assuming a single rate-limited process: $k=A^{*} \exp \left(-E_{d} / k_{B} T\right)$, where $E_{d}$ is a localized state deactivation energy (binding energy) and $A$ is a proportionality coefficient, the experimental data can be well fitted (Fig. 4). The obtained values for SOI nanodots are $E_{d}=0.7 \mathrm{meV}$ and $A=0.38$. The statistics accumulated for an oxidized $\mathrm{NC}$ sample is worse, but it exhibits similar temperature dependence (Fig. 4, inset). Fitting with the same model yields $E_{d}=$ $3.9 \mathrm{meV}$ and $A=0.67$. So, the recombination mechanism presumably returns to the QC scenario with increasing temperature. At the same time, a reverse process within one spectrum acquisition time period can occur. Then, the luminescence spectrum switches back to the localized state-induced ZPLonly line shape. The detected signal in such a case is a mixed QC and a localized state emission, resulting in the observed random scatter of the line ratio for the spectra with a TOL. As for the difference in $E_{d}$ for differently prepared NCs, a higher degree of stress induced by the self-limiting oxidation of nanopillars can possibly lead to a stronger binding energy.
From the description above, it follows that the spectra with a TOL have contributions to the main line both from the QC and the localized states. An immobilized particle has a large effective mass, and the corresponding energy change should not be significant. ${ }^{22}$ Then, a localization-induced energy change would result in a broader linewidth for TOL spectra compared to ZPL-only ones. Although the linewidth depends on many parameters, including dynamic charge environment, we do observe that the spectra with a TOL are generally broader than ZPL-only ones at $10 \mathrm{~K}$ (Fig. 5). The average linewidth of ZPL-only spectra is 2.7 times smaller than for the spectra with a TO-phonon replica.

The interface between the Si NC's core and the embedding $\mathrm{SiO}_{2}$ matrix is more complicated than a planar $\mathrm{Si} / \mathrm{SiO}_{2}$ interface. There are transition regions of amorphous $\mathrm{Si}$ and stressed $\mathrm{SiO}_{2}$ in between them. ${ }^{23}$ Thus, most likely, it is the interface that provides physical sites for the exciton spatial localization. Indeed, it was calculated that a no-phonon transition probability is enhanced by 2 orders of magnitude for a carrier localized at the strained interface region of $\mathrm{Si}$ NCs. ${ }^{24}$ The co-involvement of QC and localized states was also observed recently, when the degree of exciton localization in Si NCs was investigated by applying a magnetic field. ${ }^{25}$ Mixed QC and localized state-related transitions were found for as-prepared samples, while the QC effect dominated in a passivated specimen.

An important practical implication of the reported localized state-mediated quasidirect transition is a possible stronger recombination rate in $\mathrm{Si}$ NCs. From the present data, it is difficult to extract this parameter because of the inherent on/off blinking effect. ${ }^{26}$ Luminescence intensity in the on state at various temperatures should be extracted and should be compared in future experiments.

\section{CONCLUSIONS}

In brief, based on low-temperature PL measurements of differently prepared single Si NCs, a different type of quasidirect transition in indirect band-gap NCs was identified. Its behavior differs from the QC model prediction, implying localized state involvement in the recombination process. The presence of an additional recombination channel suggests a decrease in the radiative lifetime can be achieved in such nanostructures through appropriate interface engineering.

\section{ACKNOWLEDGMENTS}

This work was supported by the Ministry of Education, Science, Sports and Culture (MEXT) through a Grant-in-Aid for Scientific Research Grant No. 21560036 and "Nanotechnology Network Project." The authors also acknowledge support through the research centre LC510 and the Research Plan No. MSM 0021620835 (J. V.) as well as from the Swedish Research Counsel (VR) (J. L.).
${ }^{1}$ P. J. Dean, J. R. Haynes, and W. F. Flood, Phys. Rev. 161, 711 (1967).

\footnotetext{
${ }^{2}$ A. W. Fang, H. Park, O. Cohen, R. Jones, M. J. Paniccia, and J. E. Bowers, Opt. Express 14, 9203 (2006).
} 
${ }^{3}$ J. Liu, X. Sun, D. Pan, X. Wang, L. C. Kimmerling, T. L. Koch, and J. Michel, Opt. Express 15, 11272 (2007).

${ }^{4}$ W. D. A. M. de Boer, D. Timmerman, K. Dohnalova, I. N. Yassievich, H. Zhang, W. J. Buma, and T. Gregorkiewicz, Nat. Nanotechnol. 5, 878 (2010).

${ }^{5}$ M. S. Hybertsen, Phys. Rev. Lett. 72, 1514 (1994).

${ }^{6}$ D. Kovalev, H. Heckler, M. Ben-Chorin, G. Polisski, M. Schwartzkopff, and F. Koch, Phys. Rev. Lett. 81, 2803 (1998).

${ }^{7}$ I. Sychugov, R. Juhasz, J. Valenta, and J. Linnros, Phys. Rev. Lett. 94, 087405 (2005).

${ }^{8}$ I. Sychugov, Y. Nakayama, and K. Mitsuishi, Nanotechnology 21, 285307 (2010).

${ }^{9}$ J. Valenta, A. Fucikova, F. Vacha, F. Adamec, J. Humpolickova, M. Hof, I. Pelant, K. Kusova, K. Dohvalova, and J. Linnros, Adv. Funct. Mater. 18, 2666 (2008).

${ }^{10}$ I. Sychugov, Y. Nakayama, and K. Mitsuishi, J. Phys. Chem. B 113, 21516 (2009).

${ }^{11}$ H. I. Liu, N. I. Maluf, R. F. W. Pease, D. K. Biegelsen, N. M. Johnson, and F. A. Ponce, J. Vac. Sci. Technol. B 10, 2846 (1992).

${ }^{12}$ N. Pauc, V. Calvo, J. Eymery, F. Fournel, and N. Magnea, Phys. Rev B 72, 205325 (2005).

${ }^{13} \mathrm{~S}$. V. Gaponenko in Optical Properties of Semiconductor Nanocrystals (Cambridge University Press, Cambridge, UK, 1998), pp. 111-117.

${ }^{14}$ S. A. Empedocles and M. G. Bawendi, J. Phys. Chem. B 103, 1826 (1999).
${ }^{15}$ M. Fujii, Y. Kanzawa, S. Hayashi, and K. Yamamoto, Phys. Rev. B 54, R8373 (1996).

${ }^{16}$ T. Takagahara, J. Lumin. 70, 129 (1996).

${ }^{17}$ B. Bruhn, J. Valenta, and J. Linnros, Nanotechnology 20, 505301 (2009).

${ }^{18}$ P. Y. Yu and M. Cardona, Fundamentals of Semiconductors (Springer-Verlag, Berlin, 2001), p. 53.

${ }^{19} \mathrm{P}$. Y. Yu and M. Cardona, Fundamentals of Semiconductors (Springer-Verlag, Berlin, 2001), pp. 471-472; S. V. Gaponenko Optical Properties of Semiconductor Nanocrystals (Cambridge University Press, Cambridge, UK, 1998) pp. 3-4.

${ }^{20}$ H. Richter, Z. P. Wang, and L. Ley, Solid State Commun. 39, 625 (1981).

${ }^{21}$ G. Davies, J. Lumin. 80, 1 (1999).

${ }^{22}$ M. Nirmal, C. B. Murray, and M. G. Bawendi, Phys. Rev. B 50, 2293 (1994).

${ }^{23}$ N. Daldosso, M. Luppi, S. Ossicini, E. Degoli, R. Magri, G. Dalba, P. Fornasini, R. Grisenti, F. Rocca, L. Pavesi, S. Boninelli, F. Priolo, C. Spinella, and F. Iacona, Phys. Rev. B 68, 085327 (2003).

${ }^{24}$ C. Delerue, G. Allan, and M. Lannoo, Phys. Rev. B 64, 193402 (2001).

${ }^{25}$ S. Godefroo, M. Hayne, M. Jivanescu, A. Stemans, M. Zacharias, O. I. Lebedev, G. van Tendeloo, and V. V. Moshchalkov, Nat. Nanotechnol. 3, 174 (2008).

${ }^{26}$ I. Sychugov, R. Juhasz, J. Linnros, and J. Valenta, Phys. Rev. B 71, 115331 (2005). 